\title{
Predictive thermodynamic models and the challenges of bioprocesses
}

\author{
J.C de Hemptinne, and R. Lugo \\ IFP Energies Nouvelles, Rueil Malmaison, France
}

Chemical engineering and petroleum engineering contain many beautiful examples of the success of phase equilibrium thermodynamic, based on the corresponding states principle, corrected by some additional parameter or otherwise using liquid phase non-ideality (activity coefficient) models. The development of thermodynamic models in this context has reached a maturity that allow describing complex mixture behaviour, thanks to predictive parametrization methods. Even complex petroleum mixtures can be described using empirical approaches, either based on volatility curves or on detailed analytical data followed by a lumping procedure.

Today, a new dimension has opened considering the complexity of mixtures originating from biomass: the ancient approaches can no longer be applied to molecules containing several polar or hydrogen-bonding groups. A stronger physical foundation is needed for the models to be developed. Several proposals have been made in the literature. In this presentation, we shall focus on some examples using different types of approaches. The SAFT (Statistical Associating Fluid Theory) family of equations of state with its group contribution allows taking into account the different types of molecular interactions. The COSMO family will also be discussed briefly as it allows making the link with $a b$ Initio information and as such it provides a truly predictive tool. Finally, some examples of applications will be presented. 